\title{
The Potential Effect of the IDH1 Mutation and MGMT Gene Promotor Methylation on the Control of Glioblastoma-Associated Epilepsy in Patients Receiving Anti-Epileptic Agents and Chemotherapies
}

Maher Kurdi ( $\triangle$ Ahkurdi@kau.edu.sa )

King Abdulaziz University https://orcid.org/0000-0002-8979-3849

Nadeem Shafique Butt

King Abdulaziz University

\section{Saleh Baeesa}

King Abdulaziz University

\section{Badrah Alghamdi}

King Abdulaziz University

Yazid Maghrabi

King Faisal Specialist Hospital and Research Centre - Jeddah

Anas Bardeesi

King Faisal Specialist Hospital and Research Center

Rothaina Saeedi

King Abdulaziz University

Fahad Alghamdi

King Abdulaziz University

Najla Alghanmi

King Abdulaziz University

Mohammed O. Bari

King Abdulaziz University

Alaa Samkari

King Saud bin Abdulaziz University for Health Sciences

Ahmed I. Lary

King Saud bin Abdulaziz University for Health Sciences

Taghreed Alsinani

King Fahad General Hospital 
Keywords: Glioblastoma, Epilepsy, MGMT promotor methylation, IDH1 mutation, Anti-epileptic drugs, Temozolomide

Posted Date: March 17th, 2021

DOI: https://doi.org/10.21203/rs.3.rs-314962/v1

License: (c) (1) This work is licensed under a Creative Commons Attribution 4.0 International License. Read Full License 


\section{Abstract}

Objective: (a) To assess the control of glioblastoma-associated seizure in patients receiving different antiepileptic drugs and chemotherapies after total resection and its association with 0-methylguanine-DNA methyltransferase (MGMT) promotor methylation and the isocitrate dehydrogenase 1 (IDH1) mutation; (b) to determine which anti-epileptic drug exerts the best effective control on glioblastoma-associated epilepsy; (c) to identify the relationship between seizure control and different anti-epileptic drugs and recurrence interval.

Methods: This was a retrospective cohort study of patients with postoperative glioblastoma-associated epilepsy in the period between 2014 and 2019. The correlations between the IDH1 mutation and MGMT promotor methylation with anti-epileptic drugs, chemotherapy type, seizure control, and recurrence interval were analyzed using different statistical methods.

Results: This study included 53 adult patients with glioblastoma-associated epilepsy. The IDH1 mutation was present in 20 patients, and MGMT promotor methylation was present in 13 patients. Out of 53 patients, 37 cases received chemoradiotherapy while 16 cases receive only radiotherapy. Levetiracetam was the most prescribed anti-epileptic drug $(n=36,60 \%)$, and 36 and 16 patients had controlled and uncontrolled seizures, respectively. The IDH1 mutation and unmethylated MGMT promotor were significantly present in cases with controlled epilepsy $(P<0.05)$. Among the anti-epileptic drugs, levetiracetam showed significantly better seizure control in cases with the IDH1 mutation and unmethylated MGMT promotor $(P<0.05)$. Patients did not show significant differences in either seizure control or the tumor recurrence interval $(P>0.05)$.

Conclusion: (a) Glioblastoma-associated epilepsy can be better controlled in patients with the IDH1 mutation and unmethylated MGMT promotor compared with patients with the methylated MGMT promotor, (b) Levetiracetam is considered the first-line anti-epileptic drug for controlling seizures but may not enhance the sensitivity of glioblastomas to chemotherapies, (c) Lack of seizure control in glioblastoma patients may not be related to tumor recurrence despite one-year treatment with antiepileptic drugs, (d) Better understanding of the risk factors and mechanisms associated with gliomaassociated epilepsy are needed to improve patient quality of life.

\section{Introduction}

Glioblastoma-associated epilepsy is an uncommon manifestation of infiltrative gliomas, and approximately $30 \%$ of cases are drug-resistant even after glioma resection (1) (2) (3). The cause of this is still unclear; however, it is likely that genetic factors of the peritumoral brain tissue are responsible for these seizures (1) (4) (5) (6). There is evidence that tumor growth may stimulate the epileptic focus, suggesting that the two conditions may share common pathogenic mechanisms and influence each other (5). Another possible explanation is the association of gliomas with the isocitrate dehydrogenase 1 (IDH1) mutation. Previous studies showed that IDH1-mutant gliomas are more likely to cause seizures 
than IDH1-wildtype (wt) gliomas (6) (7) (8), as the mutant enzyme reduces a-ketoglutarate to D-2hydroxyglutarate. Hence, D-2-hydroxyglutarate overproduction and its structural similarity to glutamate have been suggested to play a role in the neuronal excitation leading to seizures (6). While the association of the IDH1 mutation with preoperative seizures has been previously reported, the influence of the IDH1 mutation in the postoperative glioma microenvironment is yet to be clarified. Multimodal strategies fail to control seizures (9), and a better understanding of the risk factors and mechanisms of glioma-associated epilepsy are needed to improve patient quality of life.

Gliomas with 06-methylguanine-DNA methyltransferase (MGMT) gene promoter methylation are more treatment-sensitive to temozolomide (TMZ) chemotherapy (10). However, the association of gliomaassociated epilepsy with MGMT promotor methylation has not been extensively studied. Patients with MGMT methylated gliomas show more seizure control than those with non-methylated MGMT(11) (8). In this study, we assessed seizure control among glioblastoma patients, after total resection, receiving different anti-epileptic drugs and chemotherapies and investigated its associations with MGMT promotor methylation and the IDH1 mutation. Levetiracetam is the most potent $M G M T$ inhibitor among several anti-epileptic medications with diverse $M G M T$ regulatory actions and is considered the first-line drug to control seizures in glioma patients (12). Multiple studies concluded that levetiracetam and valproic acid are the main anti-epileptic drugs that exhibit antitumor effects and decrease the mortality rate among glioblastoma patients with epilepsy (13) (14) (15). In this study, we also have investigated which antiepileptic drug exerts the best effective control of glioblastoma-associated epilepsy in Saudi Arabia and have also identified the relationship of seizure control with different anti-epileptic drugs and the recurrence interval. The only limitations we faced in this current study were the limited number of glioblastoma-associated seizure and the unknown biological cause of seizure progression or patient death when they showed late recurrence.

\section{Materials And Methods}

\section{Patient stratification}

This study included 53 patients with glioblastoma-associated epilepsy from two medical hospitals in Saudi Arabia in the period between 2014 and 2019.

This study was ethically approved by the National Biomedical Ethics Committee at King Abdulaziz University (HA-02-J-008).

All patients underwent total surgical resection of the tumor followed by a standard protocol of adjuvant therapies. The histological diagnosis was made according to the classification of the World Health Organization (WHO). Clinical data were retrieved from hospital records, including patients' age at diagnosis, gender, postoperative adjuvant therapies, anti-epileptic medications, history of seizure control, and MGMT methylation profile. The patients were stratified into different groups based on the types of chemotherapies and anti-epileptic drugs. Standard radiotherapy with a total dose of 60 Gy and TZM (150-200 $\mathrm{mg} / \mathrm{m}^{2}$ for $6-12$ cycles) were administered to all patients at the time of management. 
Unfortunately, all patients who had recurrence have died. Patients who showed no recurrence for more than year eventually passed away because of other comorbidities.

\section{Tumor samples}

Archival routine formalin-fixed and paraffin-embedded tumor tissues were collected from 53 patients diagnosed with glioblastoma-associated epilepsy. Hematoxylin-and-eosin-stained sections were reexamined by a certified neuropathologist (MK) to assure the histopathological diagnosis followed the WHO's classification. Unstained positive-charged slides from 46 tissue blocks were prepared for IDH1 immunostaining.

\section{Immunohistochemistry for IDH1}

To qualitatively identify wt and mutant IDH1 in tissue sections on an automated slide stainer, the mouse monoclonal antibody anti-IDH1 R132H (clone H09) and OptiView detection kit from Ventana on a BenchMark XT automated staining system were used. The assay procedure consisted of deparaffinization with EZ Prep at $75^{\circ} \mathrm{C}$, heat pre-treatment with Cell Conditioner for 68 min, and incubation with 1:20-1:50 diluted antibody for $32 \mathrm{~min}$ at $37^{\circ} \mathrm{C}$. The slides were counterstained with Hematoxylin II for $16 \mathrm{~min}$, and bluing reagent was used for $16 \mathrm{~min}$. Afterwards, the slides were removed from the slide stainer and then immersed into successive alcohol buffers at different concentrations for 3 min. Sections in which $>10 \%$ of tumor cells were positively stained were defined as IDH1-mutant (Fig. 1).

\section{Statistical analysis}

Data was described as frequencies and percentages. The recurrence interval was calculated from the time of adjuvant therapy initiation after surgical resection until the first day of tumor recurrence. The Pearson's Chi-Square test was used to explore the associations between the IDH1 mutation, MGMT promotor methylation status, seizure control, anti-epileptic drug, and chemotherapy with various study factors. Kaplan-Meier curves and log-rank tests were used to compare the distribution of recurrence times. All statistical analyses in this study were performed using IBM SPSS1 ver. 24 statistical software programs (SPSS Inc., Chicago, IL).

\section{Results}

\section{Descriptive analysis}

In total, 53 postoperative patients with glioblastoma-associated seizures were included in this study (36 men and 17 women). The mean age was 51 years. The predominant locations were the frontal $(n=21$, $39.6 \%)$ and temporal lobes $(n=17,32.1 \%)$. We observed the IDH1 mutation in 20 patients $(37.7 \%), M G M T$ methylation in 13 patients, and the unmethylated MGMT promotor in 40 patients. Furthermore, 37 patients received a standard treatment protocol of chemoradiotherapy, whereas 16 patients received only radiotherapy. Of those who received chemotherapy, 29 (78.4\%) patients received TMZ alone, and 8 (21.6\%) patients received TMZ together with other chemotherapies. Epilepsy was controlled in 36 patients 
(69.2\%), and 16 patients (30.8\%) had uncontrolled epilepsy. Table 1 and Fig. 2 summarize the descriptive distribution of the data and the study plan.

\section{Statistical analysis}

2.1 The IDH1 mutation and MGMT methylation with seizure control

Although the $I D H 1$ mutation was significantly present in glioblastoma patients with controlled seizure ( $P$ $=0.002$; Fig. 3a-b), there was no significant difference in seizure control between cases with mutant or wt IDH1 $(P=0.085$; Table 2). However, the unmethylated MGMT promotor was significantly more abundant in patients with controlled seizure than in $M G M T$ methylated patients; a significant relationship in seizure control was found between different groups of $M G M T$ methylation $(P=0.037)$. Although seizure in patients with the IDH1 mutation and unmethylated $M G M T$ promotor was significantly controlled ( $P=0.002$; Fig. $3 \mathrm{c}-\mathrm{d})$, there was no significant difference in seizure control among unmethylated MGMT cases with mutant and wt IDH1.

2.2 The relationship between the IDH1 mutation and MGMT methylation with different types of antiepileptic drugs and seizure control

Regardless of the status of the IDH1 mutation and MGMT profile, there were no significant differences in seizure control among cases treated with different anti-epileptic drugs ( $P=0.737$; Table 3 ). However, levetiracetam showed significantly better seizure control in cases with the IDH1 mutation and unmethylated MGMT promotor than in cases with methylated $M G M T(P=0.002 ;$ Fig. 4). There were no significant differences in seizure control among cases with wt $I D H 1$, regardless of the MGMT profile.

2.3 The relationship between anti-epileptic drugs and chemotherapies with seizure control and the tumor recurrence interval

There was no significant relationship between chemotherapies and anti-epileptic drugs with seizure control ( $P=0.486$; Table 4). Our analysis also showed no significant difference in seizure control among cases treated with TMZ $(P=1.6)$, levetiracetam $(P=0.25)$, TMZ plus levetiracetam $(P=0.8)$, or TMZ plus phenytoin $(P=0.75)$. Moreover, there was no significant difference in the tumor recurrence interval in glioblastoma patients receiving chemotherapeutic agents with controlled or uncontrolled epilepsy $(P=$ 0.88; Table 5; Figs. 5-6).

\section{Discussion}


Secondary epilepsy is a common manifestation of infiltrative gliomas and occurs in high-grade gliomas less commonly than in low-grade gliomas; however, the reason for this is still unclear (1). Patients with temporal and frontal gliomas are more likely to experience seizures, and low-grade gliomas with oligodendroglial pathology are strongly associated with preoperative seizures (16). Approximately $30 \%$ of gliomas are drug-resistant even after resection (2) (3); however, the underlying mechanisms of this are still poorly understood. It is likely that epilepsy occurrence rates are not merely explained by peritumoral changes and that the internal relationship of genetic factors of the peritumoral brain tissue could be responsible for these seizures (4). Multiple studies suggested that epileptogenesis is actually influenced by tumor molecular genetic markers (5) (6) (1). Another study also found that tumor growth may stimulate the epileptic focus, suggesting that the two conditions may share common pathogenic mechanisms that influence each other (5).

Another possible explanation is the association of gliomas with the IDH1 mutation. Studies showed that mutant IDH1 gliomas are more likely to cause seizures than wt IDH1 (6) (7) (8). IDH1 is a metabolic enzyme located in the cytosol catalyzing the oxidative decarboxylation of isocitrate to a-ketoglutarate. The mutant enzyme reduces a-ketoglutarate to D-2-hydroxyglutarate. Hence, overproduction of D-2hydroxyglutarate and its structural similarity to glutamate may be involved in the mechanism of neuronal excitation leading to seizures (7) (Fig. 1). Exposure to exogenous D-2-hydroxyglutarate increased the duration of synchronized network burst firing, and this was subsequently blocked by a selective N-methylD-aspartate receptor (NMDA) antagonist (1).

While the association of the IDH1 mutation with preoperative seizures was extensively reported, the influence of the IDH1 mutation in the postoperative glioma microenvironment is yet to be clarified. Chen et al. found that seizures are more likely to be present in low-grade gliomas with the IDH1 mutation and 1p19q codeletion than in high-grade gliomas with the IDH1 mutation. However, epilepsy during the end-oflife phase in glioblastoma patients became uncontrolled (17). Yang et al. studied the relationship of 172 gliomas with epilepsy (18) and found that $27 \%$ of glioblastomas were associated with epilepsy but that $64 \%$ of these epilepsy patients had low-grade gliomas. Furthermore, IDH1-mutant gliomas were associated with preoperative seizure mainly in cases of grade II gliomas (73\%) but not glioblastomas. Our current study also found that the IDH1 mutation is significantly associated with controlled epilepsy in glioblastoma patients.

Currently, multimodal strategies fail to control seizures in up to a third of patients (9). Thus, a better understanding of the risk factors and mechanisms associated with glioma-associated epilepsy are needed to improve patient quality of life. To the best of our knowledge, although gliomas with MGMT gene promoter methylation are known to be more treatment-sensitive to TMZ chemotherapy [10], the association of glioma-associated epilepsy and MGMT promotor methylation has never been extensively studied. The relationship between preoperative and postoperative seizure control, in regard to $M G M T$ gene promotor methylation status, is also limited. Patients with low expression of MGMT protein or MGMT methylated gliomas had more frequent postoperative seizure control than those with nonmethylated MGMT or high MGMT protein activity (11) (8). The observed favorable postoperative seizure 
control in those with MGMT gene promotor methylation could stem from the better response to adjuvant chemoradiation therapy in this patient population (8). Nevertheless, these findings did not clarify whether MGMT methylation was the main reason for postoperative seizure control or whether anti-epileptic drugs act as potent MGMT methylation inhibitors. The results of our current study have demonstrated that the unmethylated MGMT promotor had more significant seizure control.

Bobustuc et al. reported that levetiracetam was the most potent MGMT inhibitor among several antiepileptic medications with diverse $M G M T$ regulatory actions and is thus considered the first-line drug to control seizure in glioma patients receiving TMZ (12). This is because levetiracetam enhances $P 53-$ mediateed MGMT inhibition, which sensitizes glioblastoma cells to TMZ (17) (12). This mechanism has never been investigated thoroughly, and thus further studies are necessary.

Scattered studies found that levetiracetam and valproic acid are the main anti-epileptic drugs that exhibit antitumor effects and have shown an actual decrease in the mortality rate among glioblastoma patients with epilepsy (13) (14) (15). Valproic acid exhibits antitumor effects via inhibition of mSin3A/histone deacetylase 1 and modulation of the mitogen-activated protein kinase pathway. Additionally, owing to the radio-sensitizing properties of valproic acid, radiotherapy with valproic acid is more effective than radiotherapy with other drugs (19). Another study on 418 patients with glioblastoma-associated seizures in Korea found that levetiracetam treatment in cases with the methylated MGMT promoter positively influenced the overall survival rate. In our study, we also found that levetiracetam is the first-line drug for glioblastoma-associated epilepsy. However, we reject the idea that levetiracetam sensitizes glioblastoma cells to chemotherapy, as there was no significant relationship with tumor recurrence interval when both anti-epileptic drugs and chemotherapies were used in glioblastoma patients.

\section{Conclusion}

Our study has come to three main conclusions. First, glioblastoma-associated epilepsy can be better controlled in patients with the IDH1 mutation or unmethylated MGMT promotor than in patients with the methylated MGMT promotor. Second, levetiracetam can be considered the first-line anti-epileptic drug for controlling seizures yet may not enhance the sensitivity of glioblastomas to chemotherapies. Third, the lack of seizure control in glioblastoma patients is not related to tumor recurrence despite one-year treatment with anti-epileptic drugs. We recommend using IDH1 and the MGMT gene promotor as parameters in seizure control. We also recommend using levetiracetam as first-line treatment, regardless of the type of chemotherapeutic agent.

\section{Declarations}

\section{Note}

Unknown cases are not included in this table. Pearson's Chi-squared test ${ }^{1}$ 


\section{Note}

Unknown cases are not included in this table. Pearson's Chi-squared test ${ }^{1}$

\section{Funding:}

No funding received for the current study.

\section{Consenting}

The study did not include any patient information required consent for publications.

\section{Authors' contributions:}

MK, idea, IRB submission, writing, study design and data, and histological analysis; NS, Statistical analysis; $\mathrm{SB}$, data provider, writing, and analysis; $\mathrm{BG}$, study design, writing, and editing; $\mathrm{YM}$, data entry, tissue collection, and writing; $A B$, data entry, and tissue collection; RS, data entry, tissue collection, and writing; FA, Histological and data analysis, NA, Data collection, MB: IHC analysis and histological correlation; AS, data provider, writing, and histological revision; AL, data provider, and IRB submission; TA, Clinical information provider.

\section{Acknowledgment:}

none.

\section{References}

1. Chen $\mathrm{H}$, Judkins $\mathrm{J}$, Thomas $\mathrm{C}$, et al. Mutant IDH1 and seizures in patients with glioma. Neurology. 2017;88:1805-13.

2. Van Breemen MS, Wilms EB, Vecht CJ. Epilepsy in patients with brain tumours: epidemiology, mechanisms, and management. Lancet Neurol. 2007;6:421-30.

3. Goldstein ED, Feyissa AM. Brain tumor related-epilepsy. Neurol Neurochir Pol. 2018;52:436-435.

4. De Groot $M$, lyer $A$, Zurolo $E$, et al. Overexpression of ADK in human astrocytic tumors and peritumoral tissue is related to tumor-associated epilepsy. Epilepsia. 2012;53:58-66.

5. Huberfeld G, Vecht CJ. Seizures and gliomas: towards a single therapeutic approach. Nat Rev Neurol. 2016;12:204-16.

6. Feyissa AM, Worrell GA, Tatum WO, et al. High-frequency oscillations in awake patients undergoing brain tumor-related epilepsy surgery. Neurology. 2018;90:e1119-25. 
7. Zhong Z, Wang Z, Wang Y, You G, Jiang T. IDH1/2 mutation is associated with seizure as an initial symptom in low-grade glioma: a report of 311 Chinese adult glioma patients. Epilepsy Res. 2015;109:100-5.

8. Feyissa AM, Worrell GA, Tatum WO, Chaichana KL, et al. Potential influence of IDH1 mutation and MGMT promotor methylation on glioma-related preoperative seizures and postoperative seizure control. Seizure: Eur J Epilepsy. 2019;69:293-289.

9. Vecht CJ, Kerkhof M, Duran-Pena A. Seizure prognosis in brain tumors: new insights and evidencebased management. Oncologist. 2014;19:751-9.

10. Wick W, Weller M, van den Bent M, et al. MGMT testing: the challenges for biomarker- based glioma treatment. Nat Rev Neurol. 2014;10:372-85.

11. Yang Y, Mao Q, Wang X, Liu Y, et al. An analysis of 170 glioma patients and systemic review to investigate the association between IDH-1 mutations and preoperative glioma-related epilepsy. $\mathrm{J}$ of Clinic Neuroscience. 2016;31:56-62.

12. Bobustuc $G$, Bajer $C$, Limaye A, Jenkins W, et al. Levitirecetam enhances P53-mediated MGMT inhibition and sensitizes glioblastoma cells to temozolomide. Neurol Oncology. 2010;12(9):917-27.

13. Turkalp Z, Karamchandani J, Das S. IDH mutation in glioma: new insights and promises for the future. JAMA Neurol. 2014;71:1319-25.

14. Sanson $M$, Marie $Y$, Paris $S$, et al. Isocitrate dehydrogenase 1 codon 132 mutation is an important prognostic biomarker in gliomas. J Clin Oncol. 2009;27:4150.

15. Ryu JY, Lok Min K, Chang MJ. Effect of anti-epileptic drugs on the survival of patients with glioblastoma multiform: A retrospective, single-center study. PloS one. 2019;14(12):e0225599.

16. De Robles P, Fiest KM, Frolkis AD, et al. The worldwide incidence and prevalence of primary brain tumors: a systematic review and meta-analysis. Neuro Oncol 2014; pii: nou283.

17. Pace A, Metro G, Fabi A. Supportive care in neurooncology. Curr Opin Oncol. 2010;22:621-6.

18. Yang $P$, Liang $T$, Zhang $C$, et al. Clinicopathological factors predictive of postoperative seizures in patients with gliomas. Seizure. 2016;35:93-9.

19. Shamji MF, Fric-Shamji EC, Benoit BG. Brain tumors and epilepsy: pathophysiology of peritumoral changes. Neurosurg Rev. 2009;32:275-85.

\section{Tables}

Table 1. Characteristics of patients and therapies. Isocitrate dehydrogenase 1 mutation (IDH1); Omethylguanine-DNA methyltransferase (MGMT). 


\begin{tabular}{|c|c|}
\hline & Overall $(\mathrm{N}=53)$ \\
\hline \multicolumn{2}{|l|}{ Age } \\
\hline Mean (SD) & $51.4(17.6)$ \\
\hline \multicolumn{2}{|l|}{ Gender } \\
\hline Female & $17(32.1 \%)$ \\
\hline Male & $36(67.9 \%)$ \\
\hline \multicolumn{2}{|l|}{ Tumor Location } \\
\hline Frontal & $21(39.6 \%)$ \\
\hline Occipital & $2(3.8 \%)$ \\
\hline Parietal & $13(24.5 \%)$ \\
\hline Temporal & $17(32.1 \%)$ \\
\hline \multicolumn{2}{|l|}{ Anti-Epileptic Drug } \\
\hline Carbamazepine & $1(1.9 \%)$ \\
\hline Levetiracetam & $23(43.4 \%)$ \\
\hline Levetiracetam + Carbamazepine & $2(3.8 \%)$ \\
\hline Levetiracetam + Lacosamide & $1(1.9 \%)$ \\
\hline Levetiracetam + Lamotrigine & $3(5.7 \%)$ \\
\hline Levetiracetam + Phenytoin & $5(9.4 \%)$ \\
\hline Levetiracetam + Valproic acid & $2(3.8 \%)$ \\
\hline Phenytoin & $12(22.6 \%)$ \\
\hline Others & $2(3.8 \%)$ \\
\hline Phenytoin + Lacosamide & $2(3.8 \%)$ \\
\hline \multicolumn{2}{|l|}{ Epilepsy Control Status } \\
\hline Unknown & 1 \\
\hline Controlled & $36(69.2 \%)$ \\
\hline Uncontrolled & $16(30.8 \%)$ \\
\hline \multicolumn{2}{|l|}{ IDH1 Mutation } \\
\hline IDH mutant & $20(37.7 \%)$ \\
\hline IDH wildtype & $26(49.1 \%)$ \\
\hline
\end{tabular}

Page 11/18 


\begin{tabular}{|ll|}
\hline & Overall (N=53) \\
\hline Undetermined & $7(13.2 \%)$ \\
\hline MGMT Profile & $13(24.5 \%)$ \\
\hline No MGMT hypermethylation & $40(75.5 \%)$ \\
\hline Adjuvant Therapy & \\
\hline Radiation and Chemotherapy & $37(69.8 \%)$ \\
\hline Radiotherapy & $16(30.2 \%)$ \\
\hline Chemotherapy & \\
\hline Temozolomide & $29(78.4 \%)$ \\
\hline Temozolomide plus others & $8(21.6 \%)$ \\
\hline Recurrence Status & \\
\hline No recurrence & $21(39.6 \%)$ \\
\hline Recurrence & $32(60.4 \%)$ \\
\hline
\end{tabular}

Table 2. Seizure control in cases with IDH1 mutation and MGMT promotor methylation. There were no significant differences between seizure control and IDH1 status among cases with glioblastomaassociated epilepsy. However, a significant relationship in seizure control was found between different groups of MGMT methylation.

\begin{tabular}{|cllll|}
\hline & & \multicolumn{3}{c|}{ Seizure Control Status } \\
\hline Molecular Profile & Controlled & Uncontrolled & Total $(\mathbf{n = 5 3 )}$ & P-value \\
\hline IDH1-mutant & $17.0(85.0 \%)$ & $3.0(15.0 \%)$ & $36.0(69.2 \%)$ & $0.085^{1}$ \\
\hline IDH1-wildtype & $16.0(64.0 \%)$ & $9.0(36.0 \%)$ & $16.0(30.8 \%)$ & \\
\hline Methylated MGMT & $6.0(16.7 \%)$ & $7.0(43.8 \%)$ & $13.0(25.0 \%)$ & 0.0371 \\
\hline Unmethylated MGMT & $30.0(83.3 \%)$ & $9.0(56.2 \%)$ & $39.0(75.0 \%)$ & \\
\hline
\end{tabular}

Note: Unknown cases are not included in this table. Pearson's Chi-squared test ${ }^{1}$

Table 3. The relationship of anti-epileptic drugs and seizure control, regardless of the status of the IDH1 mutation and MGMT promotor methylation. There were no significant differences in seizure control 
among cases treated with levetiracetam, phenytoin, or levetiracetam with phenytoin.

\begin{tabular}{|c|c|c|c|c|}
\hline & Controlled $(n=36)$ & Uncontrolled $(n=16)$ & Total $(n=52)$ & P-value \\
\hline Anti-Epileptic Drugs & & & & $0.737^{1}$ \\
\hline Levetiracetam & $17.0(47.2 \%)$ & $5.0(31.2 \%)$ & $22.0(42.3 \%)$ & \\
\hline Phenytoin & $8.0(22.2 \%)$ & $4.0(25.0 \%)$ & $12.0(23.1 \%)$ & \\
\hline Levetiracetam + Phenytoin & $3.0(8.3 \%)$ & $2.0(12.5 \%)$ & $5.0(9.6 \%)$ & \\
\hline Others & $8.0(22.2 \%)$ & $5.0(31.2 \%)$ & $13.0(25.0 \%)$ & \\
\hline
\end{tabular}

Pearson's Chi-squared test ${ }^{1}$

Table 4. The relationship between anti-epileptic drugs and chemotherapies with seizure control. Patients with glioblastoma-associated epilepsy, regardless of the type of anti-epileptic drugs and chemotherapies, showed no significant differences in seizure control.

\section{Seizure Control Status}

\begin{tabular}{|cllll|} 
Type of Chemotherapy & Controlled & Uncontrolled & Total & $\begin{array}{l}\text { P- } \\
\text { value }\end{array}$ \\
\hline Anti-Epileptic Drugs + Temozolomide & $\begin{array}{l}21.0 \\
(80.8 \%)\end{array}$ & $7.0(70.0 \%)$ & $28.0(77.8 \%)$ & $0.486^{1}$ \\
\hline $\begin{array}{l}\text { Anti-Epileptic Drugs + Temozolomide } \\
\text { plus Other }\end{array}$ & $\begin{array}{l}5.0 \\
(19.2 \%)\end{array}$ & $3.0(30.0 \%)$ & $8.0(22.2 \%)$ & \\
\hline
\end{tabular}

Note: Unknown cases are not included in this table. Pearson's Chi-squared test ${ }^{1}$

Table 5. The relationship between seizure control in glioblastoma patients and tumor recurrence interval. Patients with glioblastoma-associated epilepsy, regardless of the status of seizure control, showed no significant differences in the recurrence interval.

\begin{tabular}{|cllll|}
\hline & & & \multicolumn{2}{l|}{$\begin{array}{l}\text { Tumor Recurrence } \\
\text { Interval }\end{array}$} \\
\hline $\begin{array}{l}\text { Seizure Control } \\
\text { Status }\end{array}$ & $\begin{array}{l}\text { Before 1 Year } \\
(\mathbf{N}=30)\end{array}$ & $\begin{array}{l}\text { After 1 Year } \\
(\mathbf{n = 2 3})\end{array}$ & Total $(\mathbf{n = 5 3 )}$ & $\begin{array}{l}\text { P- } \\
\text { value }\end{array}$ \\
\hline Controlled & $21.0(70.0 \%)$ & $15.0(68.2 \%)$ & $36.0(69.2 \%)$ & $0.888^{1}$ \\
\hline Uncontrolled & $9.0(30.0 \%)$ & $7.0(31.8 \%)$ & $16.0(30.8 \%)$ & \\
\hline
\end{tabular}




\section{Figures}

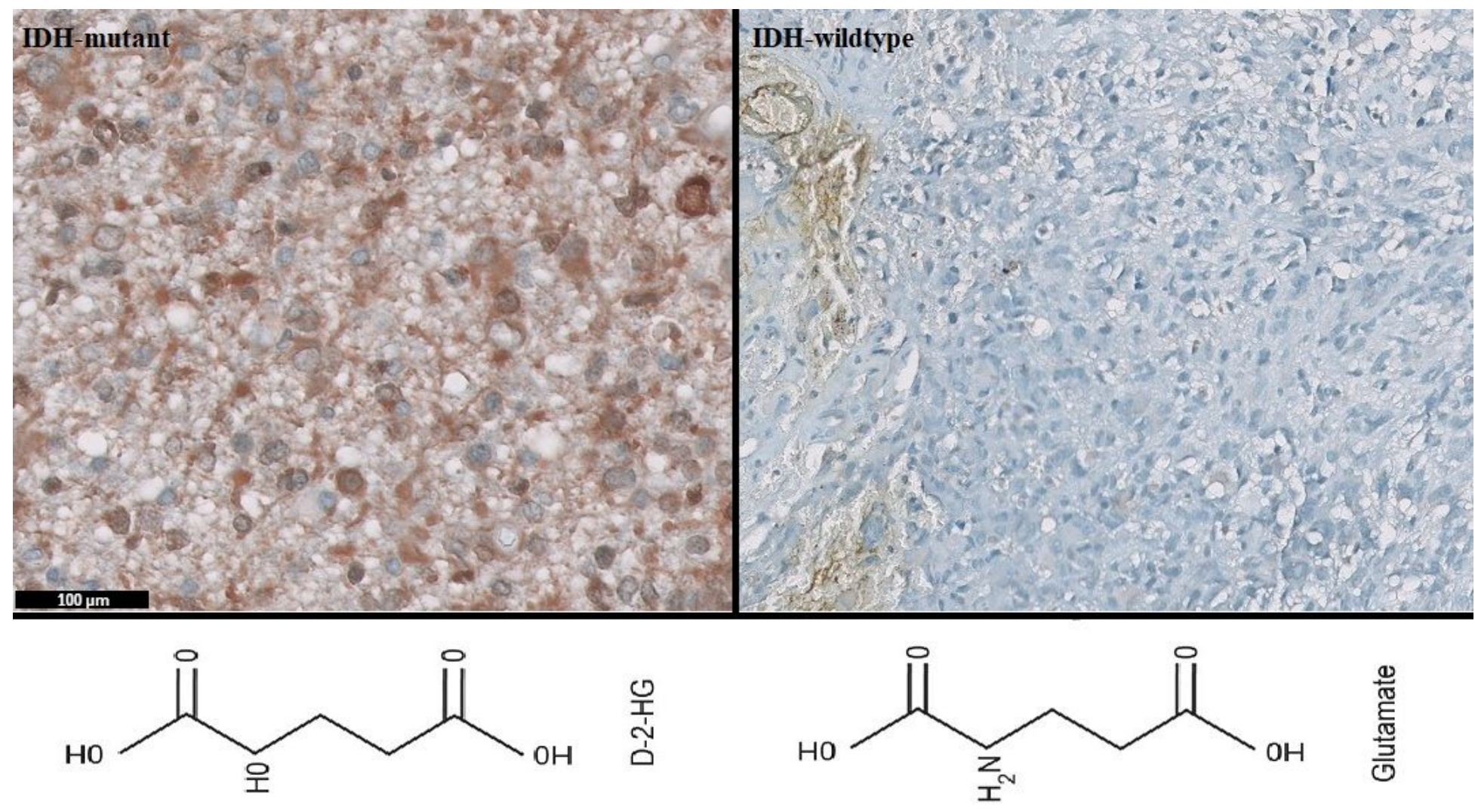

Figure 1

The expression of the IDH1 in glioblastoma-associated epilepsy. The positively expressed IDH1 mutant (left) and the negative wild-type IDH1 (right). Scale bar: $100 \mu \mathrm{m}$. 


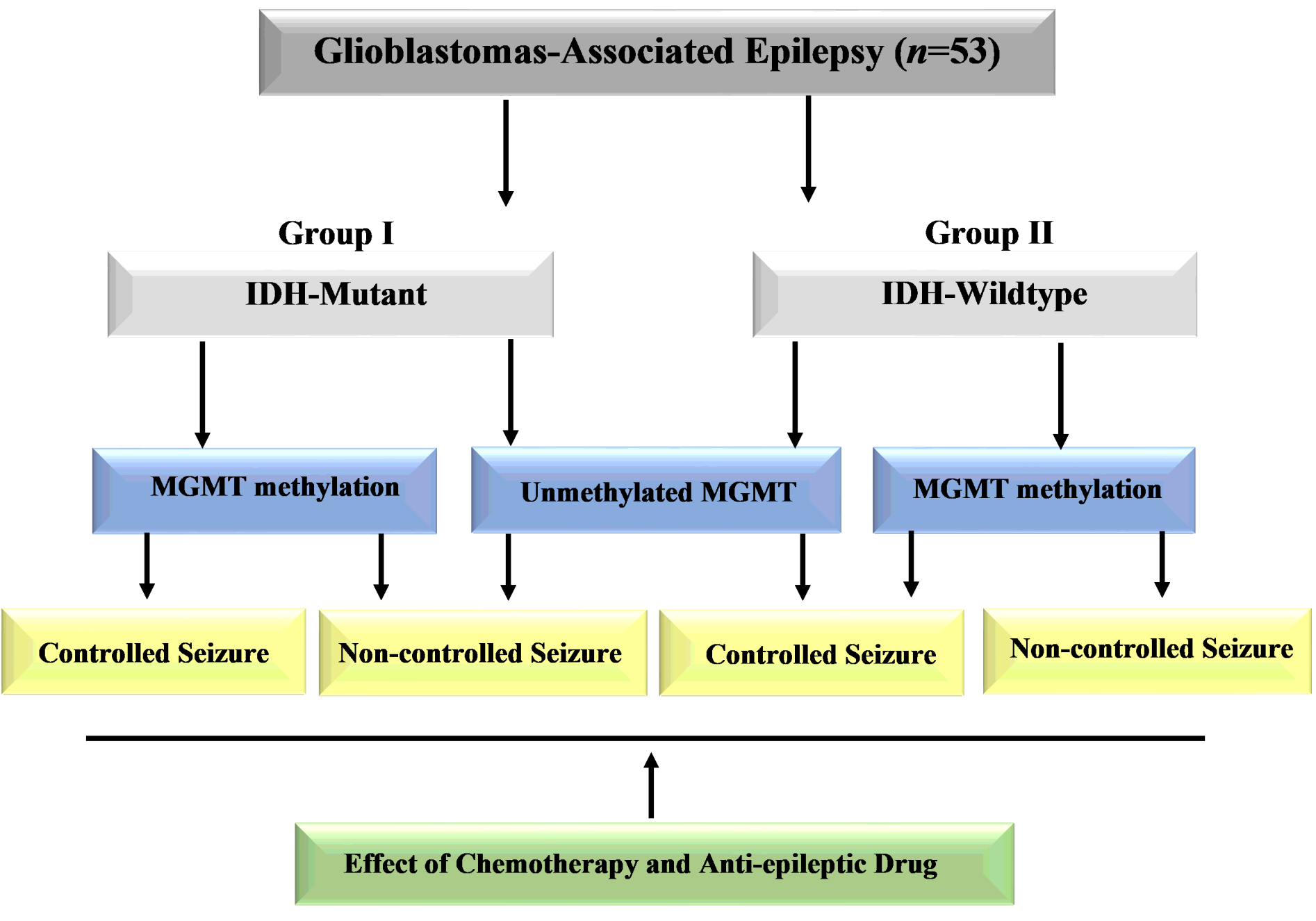

Figure 2

The algorithm of the study objectives. Cases with glioblastoma-associated epilepsy were stratified into subdivided groups based on the IDH1, 0-methylguanine-DNA methyltransferase (MGMT) methylation, and seizure control. 
a

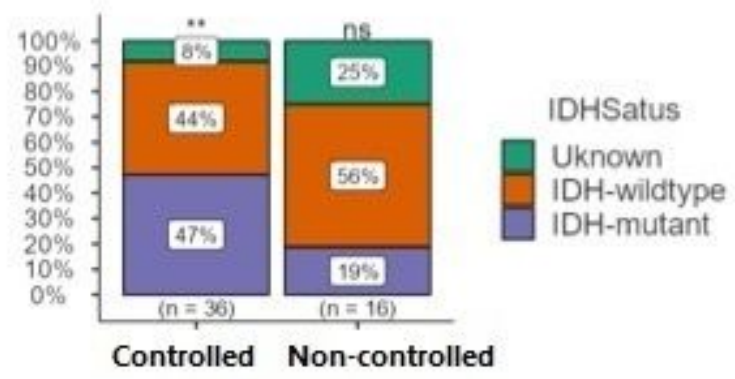

c

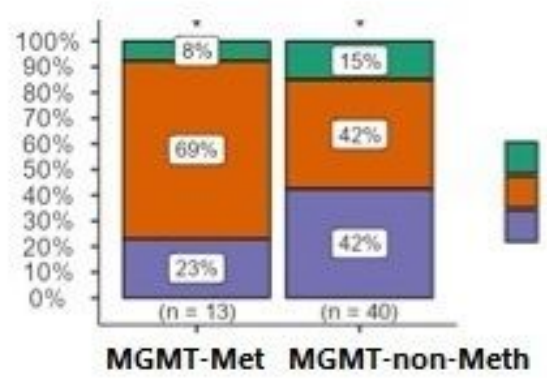

b

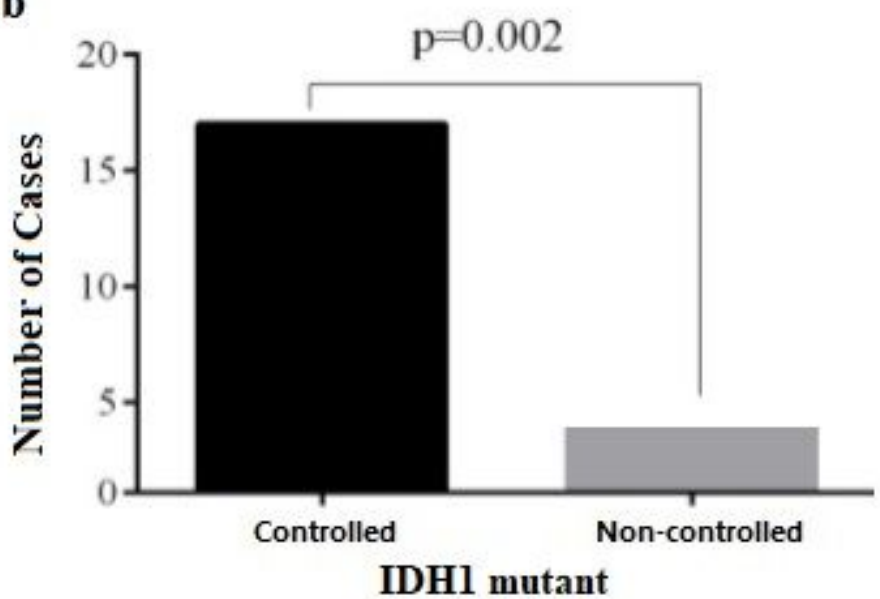

d

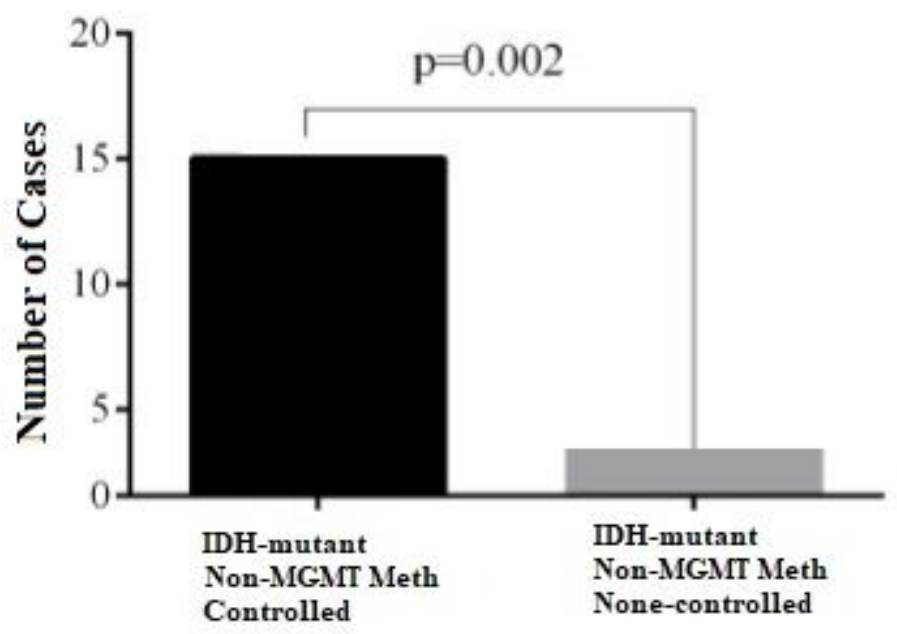

Figure 3

The IDH1 and 0-methylguanine-DNA methyltransferase (MGMT) methylation with seizure control. The IDH1 mutation is significantly present in glioblastoma patients with controlled seizure $(a, b)$. Seizure in patients with the IDH1 mutation and unmethylated MGMT promotor was significantly controlled (c,d). 


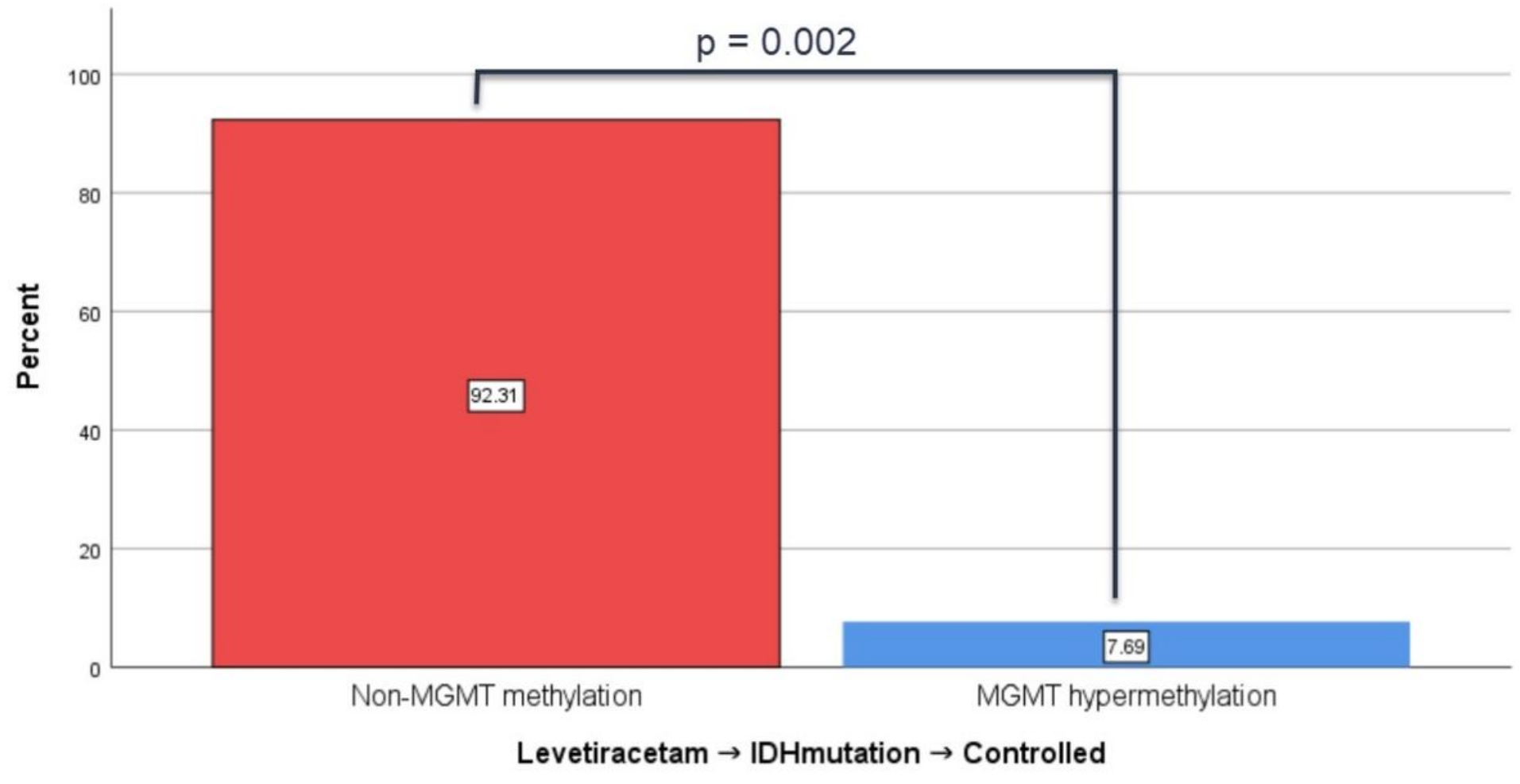

Figure 4

Levetiracetam shows significantly more seizure control in cases with the IDH1 and unmethylated Omethylguanine-DNA methyltransferase (MGMT) promotor than in cases with methylated MGMT.

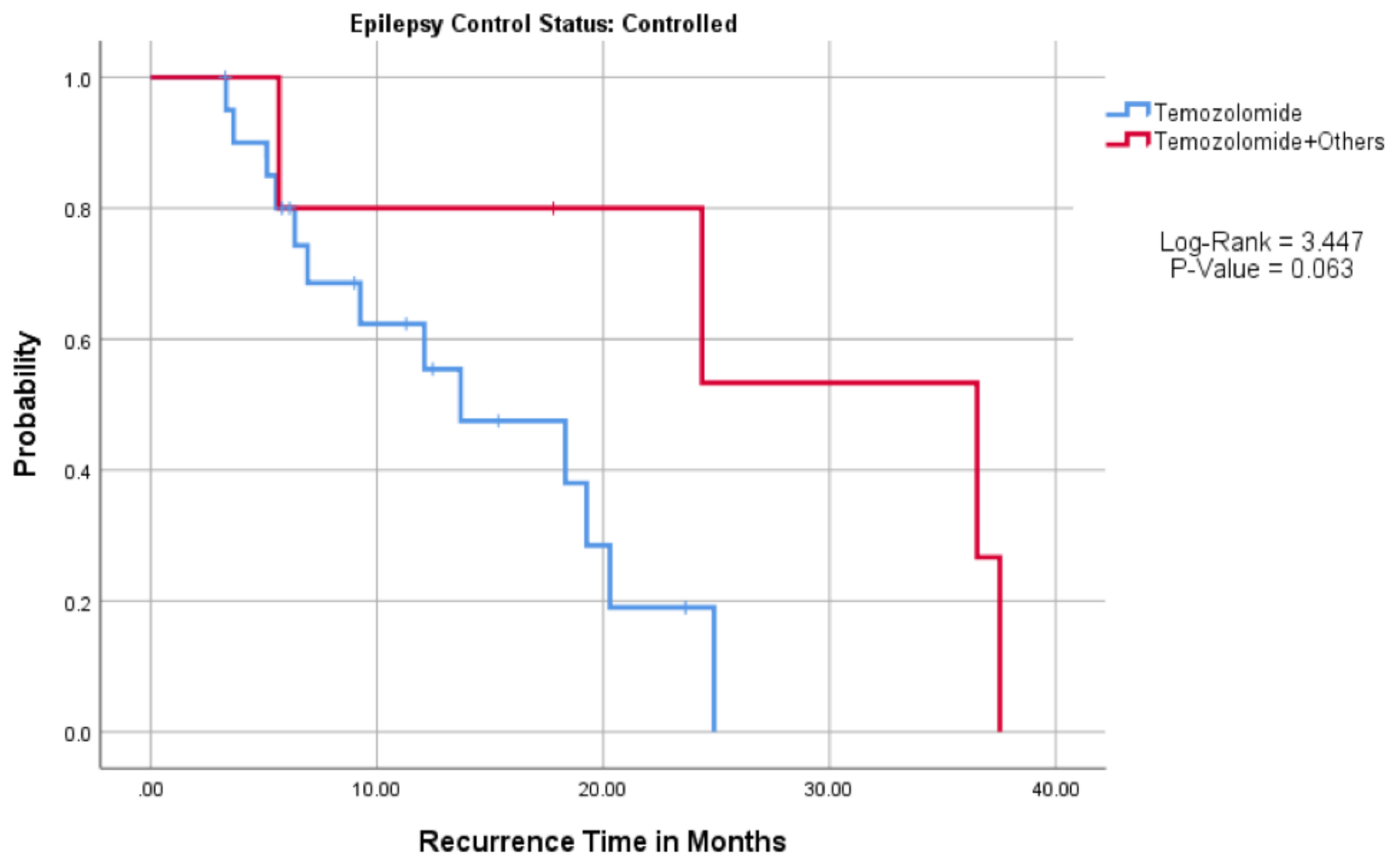




\section{Figure 5}

The relationship between seizure control and chemotherapies with the tumor recurrence interval, illustrated by Kaplan-Meier analyses. There is no significant difference in the tumor recurrence interval between glioblastoma patients with controlled epilepsy receiving chemotherapeutic agents.

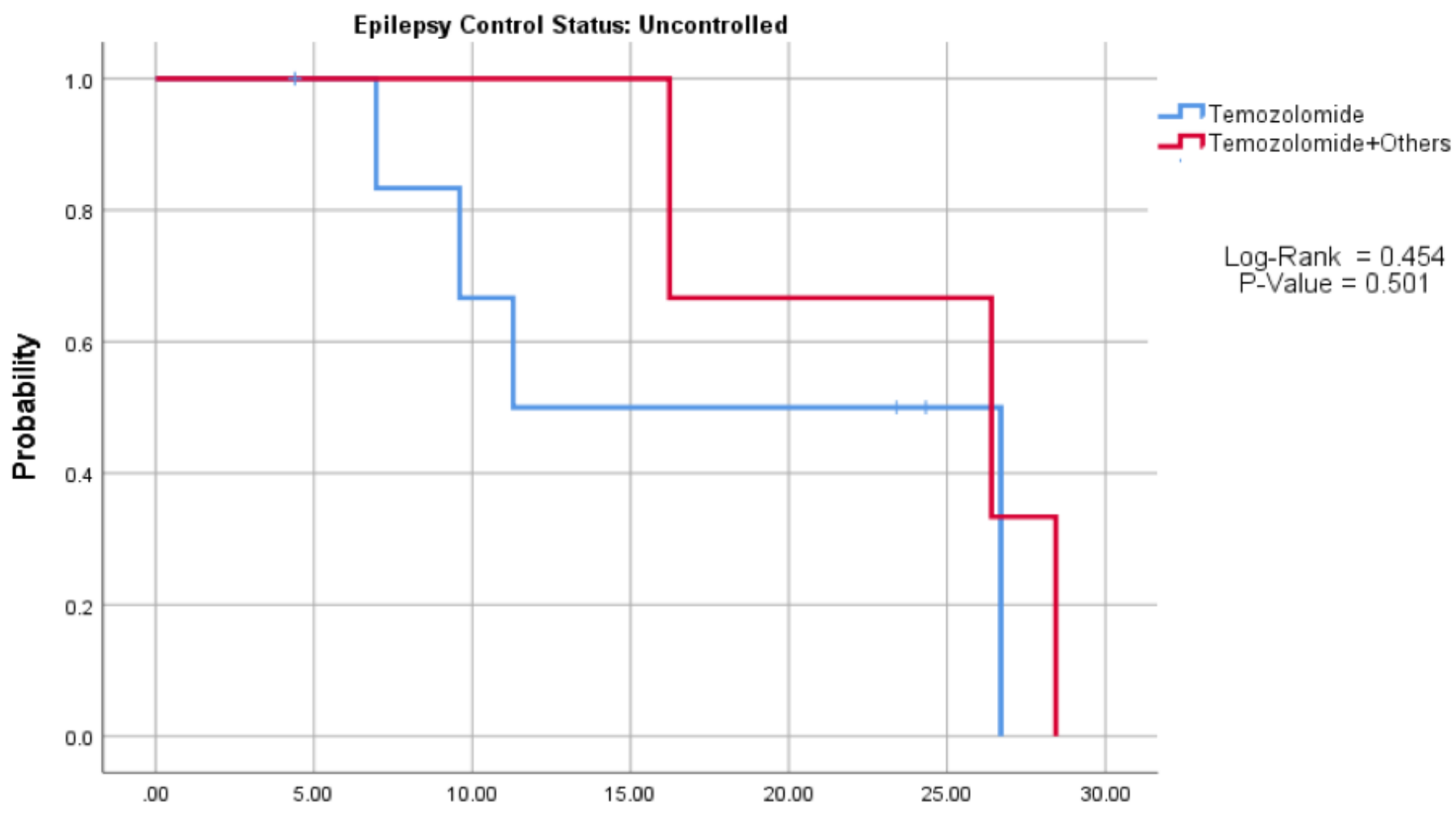

Recurrence Time in Months

\section{Figure 6}

The relationship between seizure control and chemotherapies with the tumor recurrence interval, illustrated by Kaplan-Meier analyses. There is no significant difference in the tumor recurrence interval between glioblastoma patients with uncontrolled epilepsy receiving chemotherapeutic agents. 